University of South Florida

DIGITAL COMMONS

Digital Commons @ University of

@ UNIVERSITY OF SOUTH FLORIDA

South Florida

QMaSC: A Handbook for Directors of

Quantitative and Mathematics Support Centers

USF Libraries

$1-1-2016$

\title{
06. Incorporating Technology
}

Maria Belk

Bard College

Follow this and additional works at: https://digitalcommons.usf.edu/qmasc_handbook

\section{Recommended Citation}

Maria Belk (2016), "Incorporating Technology", http://dx.doi.org/10.5038/ 9780977674435.ch6 in G. Coulombe, M. O'Neill, M. Schuckers (Eds.) A Handbook for Directors of Quantitative and Mathematical Support Centers, Neck Quill Press, http://scholarcommons.usf.edu/qmasc_handbook.

This Center Leadership and Management is brought to you for free and open access by the USF Libraries at Digital Commons @ University of South Florida. It has been accepted for inclusion in QMaSC: A Handbook for Directors of Quantitative and Mathematics Support Centers by an authorized administrator of Digital Commons @ University of South Florida. For more information, please contact digitalcommons@usf.edu. 


\section{Incorporating Technology}

(C) Maria Belk,

Bard College

प्र

\section{Introduction}

This chapter will discuss the ways in which computers and other technology can be used in a Quantitative or Mathematics Support Center (QMaSC). Section 2 will consider computer tools that can help with the administrative tasks involved in running a tutoring center, such as keeping track of the number of students who attend and getting feedback from students on how the sessions went. Sections 3 through 5 will look at computer tools that can be helpful for students and tutors during tutoring sessions.

\section{Computer Tools to Help with Running a QMaSC}

Computers can help with many of the organizational tasks involved in running a QMaSC. Here are some suggestions for using computers to help a QMaSC run more smoothly.

1. Create an online application form for tutors. An online application is easier to distribute and collect than a paper form, and the responses can be automatically stored in a spreadsheet, making it easier to look through the tutor applications. See the information on online forms below for examples of web applications that make it easy to create an online form.

Suggested Citation: Maria Belk (2016), "Incorporating Technology", http://dx.doi.org/10.5038/ 9780977674435.ch6 in G. Coulombe, M. O'Neill, M. Schuckers (Eds.) A Handbook for Directors of Quantitative and Mathematical Support Centers, Neck Quill Press, http://scholarcommons.usf.edu/qmasc_handbook.

This material is based upon work supported, in part, by the National Science Foundation under Grant DUE1255945. Any opinions, findings, and conclusions or recommendations expressed in this material are those of the author(s) and do not necessarily reflect the views of the National Science Foundation 
2. Have students sign in electronically when they enter the QMaSC. There are many advantages to having an electronic sign-in system instead of simply using paper and pen. With an electronic system, it is easier to compile data on how many students are making use of the QMaSC, and it is possible to search the data to see whether a specific student has been attending. There are commercial visitor management systems that can be used for this purpose, and there are also commercial programs such as AccuTrack or TutorTrac that include an electronic sign-in system along with many other capabilities.

3. Keep a log of activity for the QMaSC. This could include the number of students who attend, what topics they need help with, and the results of their tutoring sessions. The most obvious software to use for this would be a spreadsheet such as Excel or a spreadsheet on a cloud storage service (see below for information on cloud storage services). Alternatively, tutors could fill out an online form after each session, and the information would be automatically stored in a spreadsheet (see below for information on online forms). Commercial programs such as AccuTrack or TutorTrac can also keep track of all of this information.

4. Schedule tutor appointments online. Some QMaSC's offer individual tutoring, and computers can be helpful for scheduling these appointments. One option is to have an online form for students fill out to request tutoring appointments. Alternatively, a course management system can be used to schedule appointments (see the information below on course management systems). Commercial programs such as TutorTrac and AccuTrack also have ways to schedule appointments.

5. Create an online tutor evaluation form. An online evaluation form can be sent to students at the end of every semester, or there can be an evaluation form on the web page for the QMaSC, so that students can provide feedback throughout the semester. See the information on online forms below.

6. Share information with tutors and staff members online. For example, the tutor schedule, the contact information for all the tutors and the tutor training materials can be stored online in a cloud storage system (see below) or a course management system (see below).

7. Create an online math assessment. Some QMaSC's require students to take a math placement test or quantitative assessment. Others may find an online assessment helpful in identifying areas students need help with. Online homework systems (see below) can be used to create online assessments (in addition to online homework), and some course management systems (see below) include ways to create online quizzes or tests.

Many tools can help with these tasks: 
Cloud Storage Service: A cloud storage service such as Google Drive or Dropbox is a way to store documents online and share them with others. It can be used to share information with tutors, or simply to share files between several staff or faculty members.

- Google Drive (drive.google.com) is a free service that includes a simple web-based word processor and spreadsheet. Documents on Google Drive can be shared by many users and edited by multiple people at the same time.

- Dropbox (www.dropbox.com) is a free service for file sharing and backup for any computer. A downloadable program creates a folder on the computer in which files can be stored; the files are also accessible online and on other computers on which the Dropbox folder has been downloaded. Because the files are stored on the computer, the files can be accessed without an internet connection. It can be a problem if multiple people edit a Dropbox file at the same time; Dropbox will save both edits, but as separate files.

- Additional cloud storage services include Microsoft One Drive (https://onedrive.live. com), iWorks for iCloud (www.apple.com/iwork-for-icloud), and Amazon Cloud Drive (www.amazon.com/clouddrive). These services all work in a way similar to Dropbox.

Online Forms: Google Forms and SurveyMonkey are two web applications that make it easy to create forms that students can fill out online. Online forms can be useful for many purposes such as tutor applications, tutor evaluation forms, and requests for tutoring appointments. If a QMaSC offers one-on-one tutoring, it could use an online form for students to request one-on-one tutoring appointments. The responses would go to a spreadsheet, and someone would need to check the spreadsheet regularly and match tutors with the requests. Online forms can also be used to keep track of information about the students attending the QMaSC. The tutors can fill out a form with this information after each tutoring shift, and then the information can be stored in an online spreadsheet.

- Google Forms is one of the document types on Google Drive. The responses to a Google form are stored in a Google Drive spreadsheet, which can be downloaded as an Excel file. Spreadsheets on Google Drive can have up to 200,000 cells, so the maximum number of responses is 200,000 divided by the number of questions.

- SurveyMonkey (www.surveymonkey.com) is another website for creating online forms. It allows more question types than Google forms; however, the free version allows only 10 questions per survey and 100 responses per form. This limit on the number of responses makes it less useful for some tasks.

Course Management Systems: Most colleges and universities use a course management system 
such as Blackboard or Moodle. These systems allow the college to create web pages accessible only to certain students and faculty, such as the students enrolled in a specific course or the faculty members in a certain department. A QMaSC could have a page that is accessible only to the tutors, and the page could be used to share information with them.

Course management systems can be used to create online quizzes or tests, or to create an online math assessment. Moodle allows you to use LaTeX commands when writing quizzes, which makes it easy to include math symbols in the questions.

In addition, Blackboard and Moodle both include scheduling tools that can be used by tutors to schedule one-on-one appointments with students. Using the scheduling tool on the course web page, tutors can indicate what times they are free to meet, and then students can sign up for appointments. This system works best if each course is assigned a designated tutor who can coordinate with the professor.

Electronic Visitor Management Systems: Visitor management systems are intended for businesses to use as electronic sign-in forms for visitors to an office. At a QMaSC, software such as this can be used to keep track of which students attend. Students can sign in when they enter the QMaSC and sign out when they leave. In case a student forgets to sign out, the software can be set to sign students out automatically at the end of the day. Many visitor management systems are made for tablets (such as iPads or Android tablets) the touchscreen is convenient for an electronic sign-in form, and a tablet is less expensive than desktop computer. An example program is SIGN IN, which works on the iPad and costs a one-time fee of $\$ 54.99$ (as of October 2013). The data from SIGN IN can be exported as a .csv file for use in Excel or another spreadsheet program.

Online Homework Systems: There are a variety of online homework systems. These are intended to be used along with a course, but they can also be used to create an online math assessment. One such online homework system is WebAssign (https://webassign.net). WebAssign contains a library of pre-made problems, and it also allows instructors to design their own problems. It is free for instructors but requires a small fee for each student. Another online homework system is Maple T.A. (https://maplesoft.com/products/mapleta), which is a commercial program by the company that makes the computational algebra system Maple. Maple T.A. has pre-made math placement tests which have been approved by the Mathematical Association of America.

AccuTrack and TutorTrac: AccuTrack (www.engineerica.com/accutrack) and TutorTrac (http://www.go-redrock.com/products/tutortrac/) are commercial software packages that can keep track of information such as how many students are attending the tutoring center and what subjects they are receiving help in. TutorTrac and AccuTrack are fairly expensive, but either is probably worth the cost for a large college or university, where the tasks of keeping track of the vis- 
itors to the tutoring center and scheduling tutor appointments might otherwise be unmanageable. As an example, the following information shows how TutorTrac works (AccuTrack works similarly):

- Using TutorTrac, students sign in when they enter the tutoring center. When they sign in, the system can automatically ask them to record what class they need help with.

- When students leave the tutoring center, they sign out, and the TutorTrac can survey them on how the session went. Alternatively, it could send an e-mail to the student with the survey, so that the student can fill it out later.

- If a QMaSC offers one-on-one tutoring in addition to drop-in tutoring, TutorTrac can also be used to schedule individual appointments. Using TutorTrac, tutors who are available for one-on-one consultation can indicate which times they are free, and then students can sign up for one of the available the time slots. TutorTrac can automatically e-mail the students and the tutors a reminder about the scheduled tutoring session.

- It is easy to look up all the data that TutorTrac has stored on the students visiting the tutoring center. For example, it is possible to look up how many students attended the center over a given period, or how often a specific student attends the center.

- TutorTrac can be used with multiple tutoring centers on a campus (such as a QMaSC and a writing center).

All of the tools considered above are programs that help with collecting data, organizing data, and sharing information with others. As technology changes, some of the specific programs suggested may become obsolete; however, similar tools are likely to continue to exist, with functionality improving over time. In particular, there are likely to be an increasing number of programs for the web and for tablets that make use of the internet and cloud storage. It can be helpful to search online every few years for new tools that help with collecting and organizing data.

\section{Mathematical Review Tools}

One of the challenges faced by a QMaSC tutor is how to help students who are struggling with weak backgrounds in mathematics. Such problems can be hard for tutors to deal with although tutors are adept at helping students with homework problems, they can have difficulty creating practice problems on material from previous courses. Fortunately, there are many resources online for reviewing mathematics. Tutors can direct students to these resources, and then the students can either try learning the material on their own, or they can work through the material with the tutors' help.

The following web pages offer review materials. The tutors could be told about these web pages, and it may also be helpful to include links on the QMaSC website. 
- Khan Academy (www.khanacademy.org provides many free short videos on a wide range of topics, not all mathematical. In math, it has videos on topics ranging from arithmetic to college-level courses such as calculus, statistics, and linear algebra. The videos vary in quality, but they can be a great resource to help students review material they may have forgotten. One disadvantage of the Khan Academy is that they don't provide many exercises for students to practice with after watching the videos.

- Kuta Software (www.kutasoftware.com) sells several applications for automatically generating worksheets on algebra and calculus. Their website also boasts a large number of free, downloadable worksheets.

- Visual Calculus (archives.math.utk.edu/visual.calculus) is a website that has several tutorials, demonstrations, and drills on topics from precalculus and calculus courses. The problems in the drills have complete solutions, which can be very helpful for students trying to learn the material.

- ALEKS (Assessment and LEarning in Knowledge Spaces, www.aleks.com) is a commonly used commercial program to help with learning mathematics. It covers material from basic mathematics to precalculus, as well as statistics. ALEKS can be purchased by an individual as well as by an institution, so it could be recommended to individual students even if the college or university has not purchased ALEKS.

- Catchup Math (http://catchupmath.com) is another commercial program that helps students learn mathematics. Catchup Math focuses on mathematics taught in high school, including pre-algebra, algebra, and geometry. It can be useful for a student who needs to review these topics.

In addition to the resources listed above, many textbook publishers have online resources for use with the textbook.

\section{Other Online Resources}

In addition to those listed above, there are several other online resources useful to tutors and students. The tutors can be told about these resources, and it may also be helpful to include links on the tutoring center's web page.

Tutors and students may find it helpful to look up online a math topic that they have forgotten. Wolfram MathWorld (http://mathworld.wolfram.com) and Wikipedia (www.wikipedia.org) are useful websites for looking up math topics, including more advanced math topics.

Wolfram|Alpha (www.wolframalpha.com) is a "computational knowledge engine" created by Wolfram Research, the company that sells Mathematica; it will do mathematical computations such as solving equations and computing derivatives and integrals. Unlike a computer algebra 
system, Wolfram|Alpha has a natural-language interface, so it is easy for students to use to check their answers. It has a free version, or for a monthly fee it offers more capabilities, such as stepby-step solutions to math problems. There are also Wolfram|Alpha apps for iPads and Android tablets.

Tutor Universe (www.tutoruniverse.com) is a website where tutors are available to answer questions in many subjects, including mathematics. The tutors on Tutor Universe charge money, but it could be useful to students who are off campus or who otherwise have difficulty making it to the QMaSC.

Mathematics Stack Exchange (http://math.stackexchange.com) is a question and answer website where anyone can post a math question, and then others will respond with a detailed answer. Many of the participants on the site are mathematics professors or graduate students, and the questions range in level from precalculus to graduate-level mathematics. Students can use this website to get help with homework, and are encouraged to include a "homework" tag if they are only looking for a hint rather than full solutions.

\section{Computers in a QMaSC}

Previous sections have discussed various online tools and software packages that might be helpful for running a QMaSC. This section will consider the role of desktop computers and other technology in the tutoring center itself.

Many of the computers in the tutoring center may belong to the students themselves. Increasingly students in college have laptops - in 2010, eighty-eight percent of college students had laptops [1]. Many students bring their laptops to the QMaSC, and they also bring tablets and smartphones. They might use these devices to complete online homework assignments, to access course web pages, or to read electronic textbooks. To accommodate the students using the internet on their devices, a QMaSC should have wireless internet available. It may also be helpful to have extra power outlets, and possibly a charging station for USB devices.

Although many students have laptops, desktop computers are still useful to have in the tutoring center, for students who either do not have laptops or do not bring their laptops to the tutoring center. Also, mathematical software (such as Mathematica or Maple) that may not be available to students on their laptops can be installed on the desktop computers.

In addition to having desktop computers, some colleges have been experimenting with using tablets (such as iPads or Android tablets) in their QMaSCs. Tablets work as well as desktop computers for many web applications and can also be used as graphing calculators and as e-readers for digital versions of textbooks. As of 2013, neither Mathematica nor Maple is available for tablets - though that is likely to change in the coming years — but both companies currently offer 
downloadable apps that can run existing Mathematica or Maple files. Also, as was mentioned in Section 2, tablets can be used as electronic sign-in forms when students enter the QMaSC.

Many colleges and universities use computational software in lower-level mathematics courses, so it can be helpful to have this software installed on the desktop computers in a QMaSC. Though some software options are expensive, it may be possible to install this software on the computers in a QMaSC as part of the site license at the college or university (check with computing services). Listed below are some examples of computational software that may be available on QMaSC computers for use in lower-level mathematics courses :

- Mathematica, Maple, Matlab, and Sage are computational software often used in mathematics courses. Mathematica, Maple, and Matlab are commercial software, whereas Sage is free and open source.

- LaTeX is a program used for typesetting mathematics. It is sometimes used in advanced math courses.

- IBM SPSS (Statistical Package for the Social Sciences) and Stata are two commercial programs commonly used in statistics courses. $\mathrm{R}$ is a free program sometimes used in statistics courses.

- Microsoft Excel or other spreadsheet software is often used for data analysis and visualization in low-level mathematics courses as well as in science and economics.

Ideally, any computational software that is used in the courses at a college or university would be available on the computers in the QMaSC. If this is not possible, there could instead be regular drop-in tutoring sessions in a computer lab for students who need to learn the software or who must use the software to complete their homework.

Since students will have questions about using the computational software, it will be helpful for some of the tutors in the QMaSC to know how to use it. One solution is to determine which tutors know how to use the software and advertise this information so that students know when they can get help. Another solution is to hold a tutor training meeting devoted to learning the computational software. Even if the tutors used this software in a previous course, they may not remember how to use it very well, so they may appreciate a quick review.

Finally, a tutoring center can also benefit from certain other technology such as a computer projector and projector screen, or an interactive whiteboard. A computer projector can be useful for a tutor working with a group of students. For example, the tutor can put on the projector a list of problems for the group to work on. A projector can also be helpful during tutor training, and it can be used by students to practice presentations. An interactive white board can save material that has been written on it. This can useful during a tutoring session - information that the tutor writes on the board can be saved and given to the student, so that the student can concentrate on understanding the material and not writing it down. 


\section{Conclusions}

This chapter has considered ways in which computers and technology can be helpful for a QMaSC. There are several computer tools that can help with the administrative tasks of running a QMaSC - online forms, cloud storage services, and course management systems can be useful for collecting data and sharing information among tutors and staff members. There are also many online resources and commercial software programs to help students learn mathematics; these can be used by a student who is working with a tutor, or they can be helpful resources for when a tutor is not available. Finally, computers in a QMaSC can be used by students for a variety of tasks, including completing online homework or accessing course web pages. The computers can also contain the computational software that is used in the lower level mathematics courses at the college.

\section{Bibliography}

[1] A. Smith, L. Rainie, and K. Zickhur, "College students and technology." Pew Internet and American Life Project: http://pewinternet.org/Reports/2011/ College-students-and-technology .aspx, 2011. 